PROCEEDINGS OF THE

AMERICAN MATHEMATICAL SOCIETY

Volume 134, Number 7, Pages 1947-1953

S 0002-9939(05)08198-0

Article electronically published on December 16, 2005

\title{
COMPACT COMPOSITION OPERATORS NOT IN THE SCHATTEN CLASSES
}

\author{
MATTHEW M. JONES
}

(Communicated by Juha M. Heinonen)

To Professor J. M. Anderson on his retirement

\begin{abstract}
We provide a direct construction of the mapping $\varphi$ given by Tom Carroll and Carl Cowen in 'Compact composition operators not in the Schatten classes' (1991, in J. Operator Theory 26).
\end{abstract}

\section{INTRODUCTION}

If $\varphi$ is an analytic self-map of the unit disk $\mathbb{D}=\{z:|z|<1\}$ in the complex plane and $H$ is a Hilbert space of functions analytic in $\mathbb{D}$, we define the composition operator acting on $f \in H$ as $C_{\varphi}(f)=f \circ \varphi$. It was shown in [C] that on various specific Hilbert spaces, including the Hardy space, there are composition operators that are compact but belong to none of the Schatten classes. This answers a question of Sarason in [C].

The construction of the function $\varphi$ in $[\mathrm{CC}$ involves the Königs function and is of particular interest given the Twisted Sector Theorem (see PC] and SSS or Joel Shapiro's survey article [S] $)$. However, the geometry of the mapping function itself is difficult to understand. It is the purpose of this paper to provide a direct construction of $\varphi(\mathbb{D})$ so that $C_{\varphi}$ is compact but not in any Schatten class.

For $\alpha>-1$ we define $D_{\alpha}$ to be the weighted Dirichlet space of functions analytic in $\mathbb{D}$ for which the norm

$$
\|f\|_{\alpha}^{2}=|f(0)|^{2}+\int_{\mathbb{D}}\left|f^{\prime}(z)\right|^{2}\left(1-|z|^{2}\right)^{\alpha} d A
$$

is finite. Here $d A$ denotes normalised Lebesgue area measure on $\mathbb{D}$. Note that the Dirichlet space is $D_{0}$, the Hardy space is $D_{1}$ and the Bergman space is $D_{2}$.

If $\varphi$ maps $\mathbb{D}$ into $\mathbb{D}$, then $\varphi$ is said to have a finite angular derivative at $\zeta \in \partial \mathbb{D}$ if there is an $\eta \in \partial \mathbb{D}$ for which the quotient

$$
\frac{\varphi(z)-\eta}{z-\zeta}
$$

has finite limit as $z \rightarrow \zeta$ non-tangentially. It is known that the above limit is finite if and only if

$$
\liminf _{z \rightarrow \zeta} \frac{1-|\varphi(z)|}{1-|z|}<\infty
$$

Received by the editors February 1, 2005.

2000 Mathematics Subject Classification. Primary 30D05, 47B33; Secondary 47B10.

(C)2005 American Mathematical Society Reverts to public domain 28 years from publication 
This is the content of the Julia-Carathéodory theorem; see Theorem 1-5 in [A]. Points of $\partial \mathbb{D}$ for which $\varphi$ has a finite angular derivative are well behaved in terms of the geometry of $\varphi$; for example, if $\varphi$ has a finite angular derivative at $\zeta$, then it is conformal there, that is, arcs in $\mathbb{D}$ ending at $\zeta$ and making an angle of $\beta$ with $\partial \mathbb{D}$ are mapped onto arcs in $\varphi(\mathbb{D})$ ending at $\eta \in \partial \varphi(\mathbb{D})$ and making the same angle $\beta$ with $\partial \varphi(\mathbb{D})$. It follows that if $\varphi(\mathbb{D})$ approaches $\zeta \in \partial \mathbb{D}$ in an angle less than $\pi$, then $\varphi$ cannot have a finite angular derivative there. It was shown in MacS that, for $\varphi$ univalent, $C_{\varphi}$ is compact on $D_{\alpha}$ if and only if $\varphi$ has no finite angular derivatives.

If $T$ is a compact operator acting on a Hilbert space $H$, then $\left(T^{*} T\right)^{1 / 2}$ is a positive compact operator. It follows that the spectrum of $\left(T^{*} T\right)^{1 / 2}$ consists of countably many eigenvalues on $[0, \infty)$. If the sequence of eigenvalues of $\left(T^{*} T\right)^{1 / 2}$ lies in $\ell^{p}$, then $T$ is said to belong to the Schatten $p$-class. The Schatten $p$-classes should be seen as gradations of compactness for an operator. Each Schatten $p$-class is dense in the compact operators in the operator norm. For this reason it is of interest, given a certain class of operators, to ask whether or not there are compact operators not in any Schatten $p$-class. This was proved by Carroll and Cowen for composition operators acting on $D_{\alpha}$ for $\alpha>0$ in [CC].

To prove the result in $[\mathrm{CC}$ the authors use the fact that on certain invariant subspaces, the operator $C_{\varphi}^{*}$ is similar to a weighted unilateral shift. Recall that a backward iteration sequence is a sequence of points $z_{n}, n=1,2, \ldots$, in $\mathbb{D}$ that satisfies $\varphi\left(z_{n+1}\right)=z_{n}$. Since $C_{\varphi}^{*} K_{z}=K_{\varphi(z)}$ for $K_{z}$ the reproducing kernel for $D_{\alpha}$, the authors of [CC] construct a $\varphi$ and a backward iteration sequence $r_{n}, n=1,2, \ldots$, so that $C_{\varphi}^{*}$ restricted to the invariant subspace

$$
\bigvee_{n \geq 1} \frac{K_{r_{n}}}{\left\|K_{r_{n}}\right\|}
$$

is similar to a weighted unilateral shift with weight sequence

$$
\left(r_{n+1}-r_{n}\right) \frac{\left\|K_{r_{n}}\right\|}{\left\|K_{r_{n+1}}\right\|} \sim c\left(\frac{1-r_{n+1}}{1-r_{n}}\right)^{\alpha / 2} .
$$

The authors prove the result by constructing a $\varphi$ with no finite angular derivatives and a backward iteration sequence $r_{n}$ such that for all $p>0$

$$
\sum_{n=1}^{\infty}\left(\frac{1-r_{n+1}}{1-r_{n}}\right)^{p}=\infty
$$

This is the starting point of this paper. In $[\mathrm{CC}$ the function $\varphi$ is defined as $\varphi=\sigma^{-1}\left(e^{-1} \sigma(z)\right)$ where $\sigma$ is a univalent mapping of $\mathbb{D}$ onto a carefully defined domain $\Omega$ in $\mathbb{C}$. Since this construction gives only the geometry of the domain $\Omega$, sometimes called the Königs domain of $\varphi$, it is of interest to give a construction of the mapping $\varphi$ explicitly. The result presented in this paper does precisely that.

Theorem 1. There is a univalent map $\varphi: \mathbb{D} \rightarrow \mathbb{D}$ such that $\overline{\varphi(\mathbb{D})}$ intersects the unit circle only at the points -1 and 1 , and a backward iteration sequence $r_{n}$, $n=1,2, \ldots$, such that

$$
\begin{gathered}
\lim _{n \rightarrow \infty} \frac{1-r_{n+1}}{1-r_{n}}=0, \\
\text { for all } p>0 \quad \sum_{n=1}^{\infty}\left(\frac{1-r_{n+1}}{1-r_{n}}\right)^{p}=\infty .
\end{gathered}
$$


By the analysis in $[\mathrm{CC}$ it follows that for the mapping $\varphi$ in Theorem 1$] C_{\varphi}$ is compact on $D_{\alpha}, \alpha>0$, but is not contained in any Schatten $p$-class for $p>0$.

\section{Proof of Theorem 1}

We will construct the mapping in the infinite strip $\mathcal{S}=\{w=x+i y:|y|<\pi / 2\}$. The unit disk is conformally equivalent to $\mathcal{S}$ by the transformation

$$
w=\log \frac{1+z}{1-z}
$$

where $\log$ denotes the principle branch of the logarithm so that $z=-1$ corresponds to $w=-\infty$ and $z=1$ to $w=\infty$.

Let $\Gamma^{+}$be the polygonal line that begins at the point $(0,1 / 2)$ and passes through the points $\left(X_{n}, X_{n+1}\right), n=1,2, \ldots$, where

$$
X_{n}=\prod_{k=1}^{n} \log (k+1) .
$$

Let $\Gamma=\Gamma^{+} \cup \Gamma^{-}$where $\Gamma^{-}$is the reflection of $\Gamma^{+}$in the $y$-axis. We let $G(x)$ be the real-valued function with graph $\Gamma$.

Now let $F(x)=\log G\left(e^{x}\right)$ and $f(x)=\frac{\pi}{2} \frac{1}{F^{\prime}(x)}$ where we define

$$
F^{\prime}(x)=\lim _{\epsilon \rightarrow 0+} F^{\prime}(x-\epsilon) .
$$

Let $x>0$ be arbitrary and suppose that $n$ is chosen so that $\log X_{n}<x<\log X_{n+1}$. Then we have that $G^{\prime}\left(e^{x}\right)=\Delta X_{n+1} / \Delta X_{n}$ where $\Delta$ is the forward difference operator, $\Delta X_{n}=X_{n+1}-X_{n}$. Therefore, if $\tau \in(0,1)$ is defined so that $e^{x}=X_{n}+\tau \Delta X_{n}$, then

$$
\begin{aligned}
F^{\prime}(x) & =\frac{e^{x} G^{\prime}\left(e^{x}\right)}{G\left(e^{x}\right)}=\left(\frac{X_{n}+\tau \Delta X_{n}}{X_{n+1}+\tau \Delta X_{n+1}}\right) \frac{\Delta X_{n+1}}{\Delta X_{n}} \\
& =\left(\tau+\frac{1}{\log (n+2)-1}\right) /\left(\tau+\frac{1}{\log (n+3)-1}\right) .
\end{aligned}
$$

Hence $F^{\prime}(x)>1$ for all $x$ and $\lim _{x \rightarrow \infty} F^{\prime}(x)=1$. Therefore the domain

$$
\Omega=\{x+i y:|y|<f(x)\} \subset \mathcal{S}
$$

is well defined and by the Riemann mapping theorem there is a conformal mapping $\psi$ of $\mathcal{S}$ onto $\Omega$, such that $\psi(0)=0, \psi^{\prime}(0)>0$.

We will show that there is a backward iteration sequence $s_{n}, n=1,2 \ldots$, such that

$$
\begin{aligned}
& \lim _{n \rightarrow \infty} s_{n+1}-s_{n}=\infty, \\
& \forall p>0 \quad \sum_{n=1}^{\infty} \exp -p\left(s_{n+1}-s_{n}\right)=\infty .
\end{aligned}
$$

This is equivalent to the conclusion of the theorem with $\varphi(z)=\psi(w)$. By the symmetry of $\Omega, \psi$ is real-valued on the real axis and $\infty$ is a fixed point of $\psi$. Furthermore it follows from the Schwartz Lemma that for real $s>0, \psi(s)<s$. We may therefore find a backward iteration sequence $s_{n}$ such that $s_{n} \uparrow \infty$ as $n \rightarrow \infty$. 
Let $D$ be a domain in $\mathbb{C}$ and let $\Gamma$ denote a family of $\operatorname{arcs}$ in $D$. A metric $\rho|d z|$ is admissible for $\Gamma$ if it is non-negative in $D$ and

$$
\int_{\gamma} \rho(z)|d z| \geq 1 \quad \text { for all } \gamma \in \Gamma .
$$

The modulus of $\Gamma$ is defined as

$$
\bmod \Gamma=\inf _{\rho} \iint_{D} \rho(z)^{2} d x d y .
$$

The modulus is conformally invariant and its reciprocal is the extremal distance (see $[\mathrm{A}]$ ).

A quadrilateral $Q$ is a Jordan domain in $\mathbb{C}$ with disjoint $\operatorname{arcs} \alpha_{1}, \alpha_{2} \subset \partial Q$. The modulus of $Q$ is defined as the modulus of $\Gamma$ where $\Gamma$ consists of all rectifiable $\operatorname{arcs}$ in $Q$ that have one endpoint in $\alpha_{1}$ and one in $\alpha_{2}$. A special case is when $Q=\{x+i y: 0<x<M, 0<y<1\}$ and $\alpha_{i}, i=1,2$, are the horizontal sides of $Q$. In this case $\bmod Q=M$ (see $[\mathrm{A}]$ ).

We will need the following exact formula for the modulus.

Lemma 1. Let $0<a<b$ be arbitrary and $Q(a, b)=\Omega \cap\{x+i y \in \mathcal{S}: a<x<b\}$ with $\alpha_{i}, i=1,2$, the two components of $Q(a, b) \cap \partial \Omega$. Then

$$
\bmod Q(a, b)=\int_{a}^{b} \frac{d x}{2 f(x)} .
$$

Proof. Let $\Gamma_{0}$ consist of the $\operatorname{arcs} \gamma_{x}=\{\Re z=x\} \cap \Omega$ for $a<x<b$ and define $\rho_{0}(w)=(2 f(x))^{-1}$ where $w=x+i y$. For any $\gamma_{x} \in \Gamma_{0}$ we have that

$$
\int_{\gamma_{x}} \rho_{0}(w)|d w|=1 \text {. }
$$

If we now let $h$ be any real-valued function defined on $Q(a, b)$ with

$$
\int_{\gamma_{x}} h(w)|d w|=\int_{\gamma_{x}} h(w) d y \geq 0,
$$

then

$$
\iint_{Q(a, b)} h(w) \rho_{0}(w) d x d y \geq \frac{1}{2 f(b)} \iint_{Q(a, b)} h(w) d y d x \geq 0 .
$$

By Beurling's criterion (Theorem 4-4 in A]), it follows that $\rho_{0}$ defines an extremal metric for $Q(a, b)$ and so

$$
\bmod Q(a, b)=\iint_{Q(a, b)} \frac{d x d y}{(2 f(x))^{2}}=\int_{a}^{b} \frac{d x}{2 f(x)}
$$

as required.

We prove (11) first. This will follow if we can show that $\psi$ does not have an angular derivative at $\infty$. Results of Jenkins, Oikawa, Rodin and Warschawski (see Theorem 6 in $[\mathrm{M}]$ ) and the references therein, imply that $\psi$ has a finite angular derivative at $\infty$ if and only if

$$
\int_{0}^{\infty}\left(\frac{1}{f(x)}-\frac{2}{\pi}\right) d x
$$



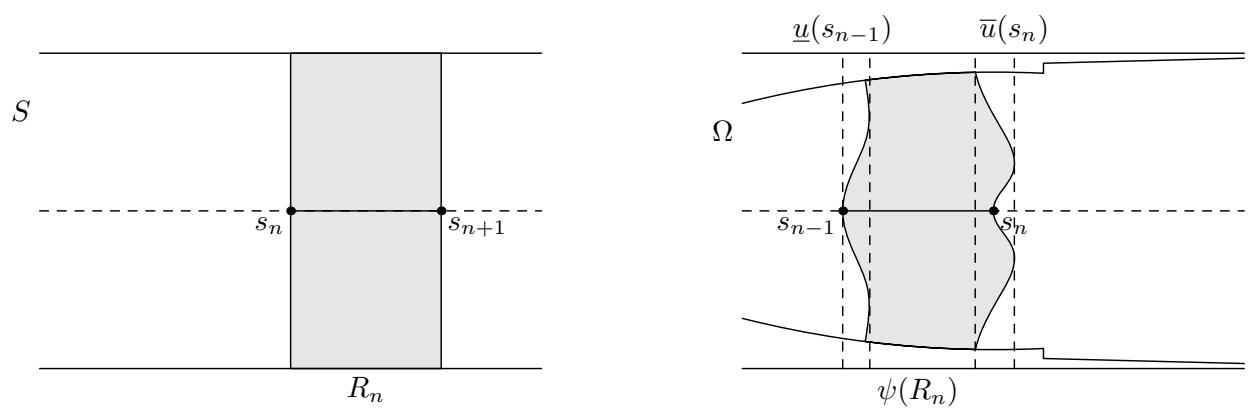

Figure 1. Proof of Lemma 2

exists. However a simple calculation shows that

$$
\int_{\log X_{n}}^{\log X_{n+1}}\left(F^{\prime}(x)-1\right) d x=\log \log (n+3)-\log \log (n+2)
$$

and so

$$
\begin{aligned}
\int_{0}^{\infty}\left(\frac{1}{f(x)}-\frac{2}{\pi}\right) d x & =\frac{2}{\pi}\left(c+\lim _{n \rightarrow \infty} \sum_{k=1}^{n} \int_{\log X_{k}}^{\log X_{k+1}}\left(F^{\prime}(x)-1\right) d x\right) \\
& =\frac{2}{\pi}\left(c+\lim _{n \rightarrow \infty} \log \log (n+3)\right)=\infty .
\end{aligned}
$$

Therefore $\psi$ does not have a finite angular derivative at $\infty$ and $s_{n+1}-s_{n} \rightarrow \infty$ as $n \rightarrow \infty$.

We now turn our attention to proving (2).

Lemma 2. The sequence $s_{n}$ asymptotically satisfies the difference equation

$$
s_{n+1}-s_{n}=\frac{\pi}{2} \int_{s_{n-1}}^{s_{n}} \frac{d x}{f(x)} .
$$

Proof. Let $R_{n}=\left[s_{n}, s_{n+1}\right] \times[-\pi / 2, \pi / 2] \subset \mathcal{S}$ and $\gamma_{n}=\mathcal{S} \cap\left\{x=s_{n}\right\}$. Note that $\psi\left(R_{n}\right)$ contains the interval $\left[s_{n-1}, s_{n}\right]$ and is symmetric about the real axis. Let $\underline{u}\left(s_{n}\right)=\inf \left\{x: x+i y \in \psi\left(\gamma_{n+1}\right)\right\}$ and similarly $\bar{u}\left(s_{n}\right)=\sup \left\{x: x+i y \in \psi\left(\gamma_{n+1}\right)\right\}$ (see Figure 1).

It then follows by the usual comparison principle of modules that

$$
\bmod Q\left(\bar{u}\left(s_{n-1}\right), \underline{u}\left(s_{n}\right)\right) \leq \bmod \psi\left(R_{n}\right) \leq \bmod Q\left(\underline{u}\left(s_{n-1}\right), \bar{u}\left(s_{n}\right)\right) .
$$

Therefore, by the conformal invariance of the modulus and Lemma 1 we have the double inequality

$$
\int_{\bar{u}\left(s_{n-1}\right)}^{\underline{u}\left(s_{n}\right)} \frac{d x}{2 f(x)} \leq \frac{s_{n+1}-s_{n}}{\pi} \leq \int_{\underline{u}\left(s_{n-1}\right)}^{\bar{u}\left(s_{n}\right)} \frac{d x}{2 f(x)} .
$$

We claim that $\bar{u}-\underline{u}$ is bounded by an absolute constant. To see this, map $Q\left(\bar{u}\left(s_{n}\right), \underline{u}\left(s_{n}\right)\right)$ into $\overline{\mathcal{S}}$ using $\psi^{-1}$. Then the image of the $\operatorname{arcs}\left\{x=\bar{u}\left(s_{n}\right)\right\} \cap \Omega$ and $\left\{x=\underline{u}\left(s_{n}\right)\right\} \cap \Omega$ both touch the line $x=s_{n+1}$ and so by the Ahlfors distortion theorem (Theorem 4-8 in [A]),

$$
\frac{\bar{u}\left(s_{n}\right)-\underline{u}\left(s_{n}\right)}{\pi} \leq \Lambda(1)=\frac{1}{2},
$$


for any $n$. Here $\Lambda(R)$ is the modulus of the Teichmüller annulus (see [A]). Therefore since $\underline{u}\left(s_{n}\right)<s_{n}<\bar{u}\left(s_{n}\right)$ we have

$$
\begin{aligned}
\left|\frac{s_{n+1}-s_{n}}{\pi}-\int_{s_{n-1}}^{s_{n}} \frac{d x}{2 f(x)}\right| & \leq \int_{s_{n-1}-\pi / 2}^{s_{n-1}} \frac{d x}{2 f(x)}+\int_{s_{n}}^{s_{n}+\pi / 2} \frac{d x}{2 f(x)} \\
& =o(1) \int_{s_{n-1}}^{s_{n}} \frac{d x}{2 f(x)}
\end{aligned}
$$

as $n \rightarrow \infty$. The last equality holds since $s_{n+1}-s_{n} \rightarrow \infty$ as $n \rightarrow \infty$.

We therefore have that the sequence $s_{n}$ is asymptotic to a sequence $x_{n}$ that satisfies

$$
x_{n+1}-x_{n}=\int_{x_{n-1}}^{x_{n}} F^{\prime}(x) d x .
$$

Lemma 3. If $x_{n}, n=1, \ldots$, is a solution to equation (4), then

$$
x_{n+1}-x_{n} \sim \log \log (n+2)
$$

as $n \rightarrow \infty$.

Proof. First note that (4) is equivalent to the equation $x_{n+1}=F\left(x_{n}\right)+c$ for a given constant $c>0$. We may assume that $c=0$, for if $x_{n}$ is a solution of the difference equation $x_{n+1}=F\left(x_{n}\right)$, then $y_{n}=x_{n}+c n$ is a solution to $y_{n+1}=F\left(y_{n}\right)+c$ and $x_{n+1}-x_{n} \sim y_{n+1}-y_{n}$ as $n \rightarrow \infty$.

Using the definition of $F$ we have

$$
\exp x_{n+1}=G\left(\exp x_{n}\right) .
$$

It now follows by definition that the sequence $X_{n}, n=1,2, \ldots$, is a solution to (4) and so

$$
\begin{aligned}
x_{n+1}-x_{n} \sim X_{n+1}-X_{n} & =\log \left(\prod_{k=1}^{n+1} \log (k+1)\right)-\log \left(\prod_{k=1}^{n} \log (k+1)\right) \\
& =\log \log (n+2) .
\end{aligned}
$$

Now since the backward iteration sequence, $s_{n}$, satisfies (3) asymptotically it follows that

$$
s_{n+1}-s_{n} \sim X_{n+1}-X_{n}=\log \log (n+2) .
$$

Therefore for any $p>0$ there is an $n_{0}$ so that for all $n>n_{0}$,

$$
\exp p\left(s_{n+1}-s_{n}\right) \leq c(\log (n))^{p}<n
$$

and so

$$
\sum_{n>n_{0}} \exp -p\left(s_{n+1}-s_{n}\right)>\sum_{n>n_{0}} \frac{1}{n}=\infty,
$$

and the theorem is proved. 


\section{REFERENCES}

[A] Ahlfors, L. V., Conformal invariants: Topics in geometric function theory, McGraw-Hill, New York (1973) MR0357743(50:10211)

[CC] Carroll, T. and Cowen, C. C., 'Compact composition operators not in the Schatten classes', J. Operator Theory 26 (1991), 109-120 MR.1214923 (94c:47045)

[C] Cowen, C. C., 'Composition operators on Hilbert spaces of analytic functions: A status report', Proceedings of Symposia in Pure Mathematics, Amer. Math. Soc. 51 (1990) part I, 131-145 MR1077383 (91m:47043)

[MacS] MacCluer, B. D. and Shapiro, J. H., 'Angular derivatives and compact composition operators on the Hardy and Bergman spaces', Canad. J. Math. 38 (1986) 878-906 MR0854144 (87h:47048)

[M] Marshall, D. E., 'Angular derivatives and Lipschitz majorants', preprint

[PC] Poggi-Corradini, P., 'Hardy spaces and twisted sectors for geometric models', Trans. Amer. Math. Soc. 348 No 6 (1996) MR1340184 (97e:30063)

[S] Shapiro, J. H., 'Composition operators and Schröder's functional equation', Contemporary Mathematics 213 (1998) 213-228 MR:1601116 (98m:47048)

[SSS] Shapiro, J. H., Smith W. and Stegenga D., 'Geometric models and compactness of composition operators', J. Funct. Anal., 127 (1995) 21-62 MR1308616 (95m:47051)

Department of Mathematics, Middlesex University, The Burroughs, Hendon, London, NW4 4BT, UNITED KINGDOM

E-mail address: m.m.jones@mdx.ac.uk 